\title{
The design of a force feedback soft gripper for tomato harvesting
}

\author{
Asiwan Kultongkham, Supakit Kumnon, Tawan Thintawornkul, Teeranoot Chanthsopeephan \\ Mechanical Engineering Department, Faculty of Engineering, King Mongkut's University of Technology Thonburi, \\ Bangkok, Thailand
}

\begin{abstract}
In smart farming, both artificial intelligence and robotic systems are applied in order to improve efficiency. In agriculture, for jobs such as seeding, monitoring, and harvesting, robots are widely used. When using robots to harvest fruit and vegetables, it is essential not to apply excessive force, as it may damage the harvest. In this paper, a soft robotic three-fingered gripper is presented. It was designed and analysed using the finite element method. Each finger is made of silicone rubber. The shape of the finger is designed so that it is capable of handling spherical shaped objects, such as tomatoes or oranges. When holding a tomato, the fingers apply the contact force. The fingers are actuated pneumatically and the force applied is also controlled by a micro controller. The pressure inside the air chamber of the finger is in the range of 0$95 \mathrm{kPa}$. Force sensors are attached to the end of each finger to provide force feedback. Then, the holding force is adjusted and applied to the surface of the tomato. The gripper can successfully grasp tomatoes with a force less than the bio-yield of the tomatoes $2.57 \mathrm{~N}$.
\end{abstract}

\section{Introduction}

In smart farming, tasks, which have been generally performed by humans, can now be covered by robots and automatic systems.

Correspondence: Teeranoot Chanthasopeephan, Mechanical Engineering Department, Faculty of Engineering, King Mongkut's University of Technology Thonburi, 126 Prachautit Road, Bangmod, Tungkru, Bangkok, 10140 Thailand.

E-mail: teeranoot.cha@kmutt.ac.th

Key words: Tomatoes harvesting; soft gripper; force-controlled gripper; smart farming

Acknowledgements and funding: this project was funded by National Research Council of Thailand (NRCT) and partially supported by the Department of Mechanical Engineering at KMUTT.

Received for publication: 16 June 2020.

Accepted for publication: 20 January 2021.

CC Copyright: the Author(s), 2021

Licensee PAGEPress, Italy

Journal of Agricultural Engineering 2021; LII:1090

doi:10.4081/jae.2021.1090

This article is distributed under the terms of the Creative Commons Attribution Noncommercial License (by-nc 4.0) which permits any noncommercial use, distribution, and reproduction in any medium, provided the original author(s) and source are credited.
Currently, the harvesting of tomatoes is done by human beings, even though advanced systems can be integrated with vision and artificial intelligence and make more efficient farming possible compared to conventional farming methods. This article presents a soft gripper which offers advantages over a typical rigid gripper, since it is more flexible and adaptable. The adaptability of a soft gripper makes it usable in many applications such as grasping objects with an irregular contour by different types of actuators i.e. pneumatic, motor, or jamming (Truby et al., 2018; Hao et al., 2020). The large contact surface of the soft gripper makes it possible to use low contact pressure, which is essential to handle delicate objects. When considering the shape of an object, it is also essential to consider the grasping force. Excessive force can cause damage to soft deformable objects i.e. fruit and vegetables. Brown et al. (2010) designed a universal jamming gripper which can be applied to hold and grasp objects of various shapes and sizes. Also a soft gripper was designed in robotics with a pneumatic actuator, which can control its motion rapidly (Mosadegh et al., 2014).

Due to the variety and physical properties of objects, packaging tasks are a challenge (Wang et al., 2017). For instance, in the lunch-box packaging process, soft grippers have performed better than other solutions. Therefore, four pneumatic soft grippers, made of different materials, were tested and compared, while experimenting with gripping a variety of objects. In order to grip objects of various materials, some prototypes were developed. Hao et al. (2020) applied a hybrid pneumatic-thermal actuator as well as a sensory feedback system in order to grasp objects. In a complex environment, feedback sensors have proven useful to allow grippers to reconfigure grasping.

As for fruit and vegetables, different gripping approaches have been applied, depending on the shape and the texture of the objects being picked (Hayashi et al., 2002; van Henten et al., 2002; Liu et al., 2007; Lehnert et al., 2017). For apple harvesting, a special apple picking robot was fabricated (Jinliang et al., 2010). Liu et al. (2013) developed a commercial Motoman manipulator integrated with a newly-designed end-effector. The hand-arm coordination proved it could easily perform the task of harvesting tomatoes with a success rate of $83.3 \%$. Moreover, a system with picking-point recognition, scissors, and gripper for picking tomatoes in a greenhouse was designed (Ji et al., 2014). An effector was also put into action to perform the task of picking tomatoes, which involved stem holding, fruit holding, and fruit separating parts (Wang et al., 2016). Two parallel plate fingers and a suction pad as well as robot grippers were developed and compared (Monta et al., 1998). Sirisomboon et al. (2012) studied the properties of Momotaro tomatoes at different stages, starting from green (raw), pink (half-ripe), and ripe (red). Hence, rupture force, bio-yield force, and penetrating force along with deformation were determined. Researchers carried out mechanical testing to calculate the bio-yield of tomatoes at different stages. The bioyield of ripe tomatoes was approximately $2.75 \mathrm{~N}$.

This paper describes the design and fabrication of a soft pneumatic gripper for tomato harvesting. The first part outlines the design process and analysis of the soft gripper. Simulations using 

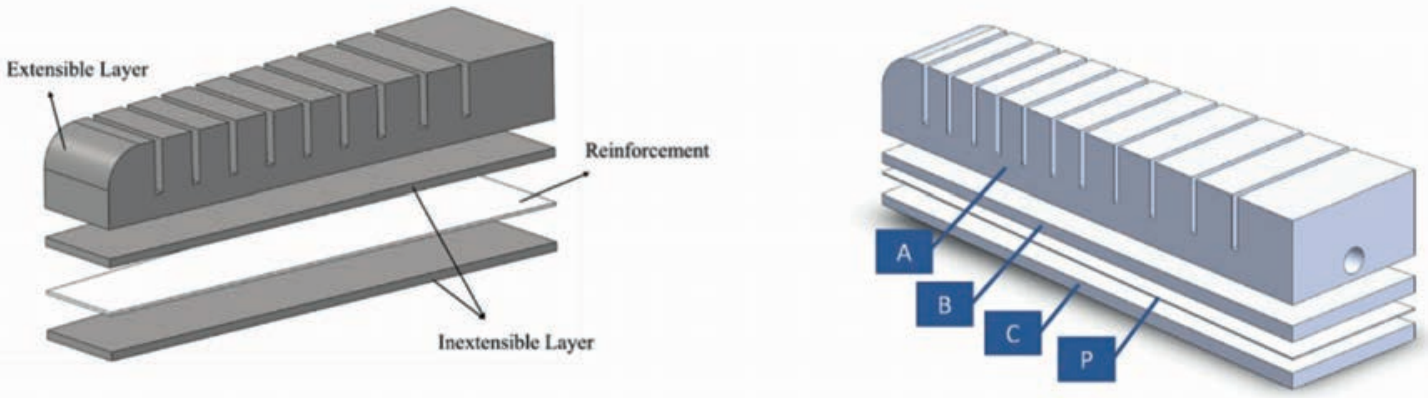

Figure 1. The finger designed with an inextensible layer, and an extensible layer.

finite element method (FEM) were conducted as part of the design process. The second part focuses on system integration with the control and feedback system and the testing of the soft gripper, highlighting the cutting and harvesting mechanism.

\section{Materials and methods}

\section{Design of the soft gripper}

The soft gripper is operated by a pneumatic system. Inside the gripper, there is an air chamber that can be inflated to actuate the finger. The gripper is made of silicone rubber and fabricated by casting a platinum-based silicone rubber SF820 into a 3D printed mould. The gripper consists of three fingers. As shown in Figure 1, each finger consists of three components: i) the soft finger containing the pneumatic chamber; ii) the base of the soft finger with piece of polycarbonate sheet layered with silicone rubber; and iii) an air tube which allows compressed air to flow in and out. Sections (A), (B) and (C) are made of silicone Sylgard 186. A polycarbonate sheet of $0.4 \mathrm{~mm}$ thickness $(\mathrm{P})$ is inserted between layers $\mathrm{B}$ and $\mathrm{C}$ to reinforce the soft gripper. The first part (A) corresponds to the air chamber. The second parts $(\mathrm{B}$ and $\mathrm{C})$ indicate the silicone layers.

The air chamber is divided into ten sub-chambers, as represented in Figure 2A where the dimensions of the sub-chambers are also provided. Each finger of the gripper is $90 \mathrm{~mm}$ long. Its layout is depicted in Figure 2B with all its relevant measurements. Figure $2 \mathrm{~A}$ presents the side view of the designed finger. There are three key parameters in this design: i) the height $(\mathrm{h})$ of the sub-chamber; ii) the side thickness (t_side) of the sub-chamber; and iii) the top thickness (t_top) of the sub-chamber. The thickness of the inextensible layer is $0.4 \mathrm{~mm}$, and the thickness of the bottom (t bottom) of the sub-chamber is $2 \mathrm{~mm}$. The height of each sub-chamber is the highest at sub-chamber \#1 and decreases gradually until sub-chamber \#10 is reached. Hence, air volume in sub-chamber \#1 is at its highest and varies throughout all the ten sub-chambers. The space between each sub-chamber is $1.5 \mathrm{~mm}$.

\section{Finite element analysis}

As part of the design process, FEM is applied to analyse the relationship between the air pressure and the deformation of the soft finger with the aim of observing the shape of the soft finger while bending. Sylgard's Mooney-Rivlin model was implemented to test the properties of the silicone to find out which property was best suited for the prototype (Bernardi et al., 2017). In Table 1, the Mooney-Rivlin model parameters and coefficients are presented
A

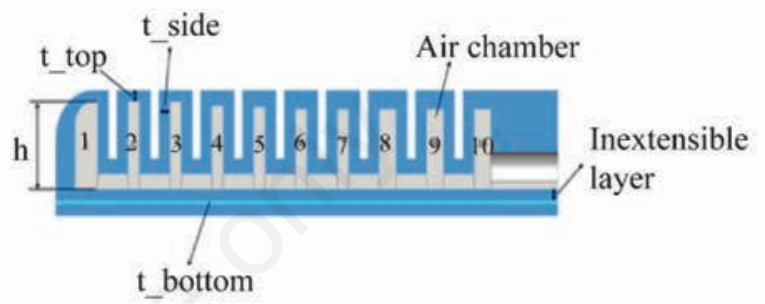

B

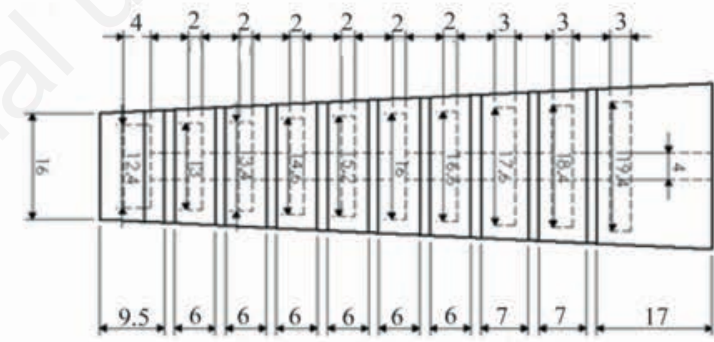

C

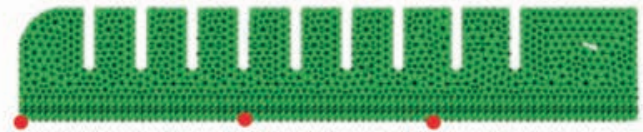

Position $1 \quad$ Position $2 \quad$ Position 3

D

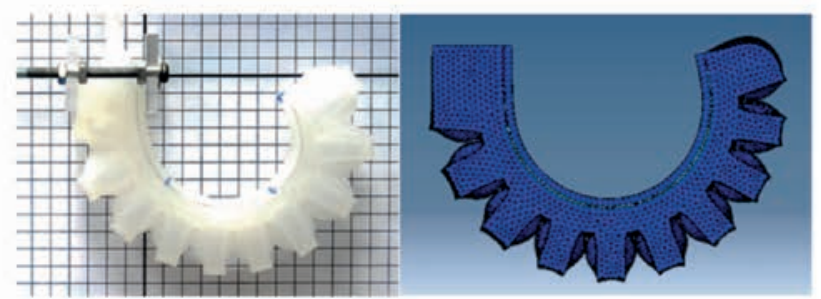

Figure 2. Dimensions of the air chamber inside the finger: (A) side view (B) top view (C) the three positions on the finger (D) the prototype and the finite element model. 
with $\mathrm{E}=2 \mu(1+v)$. Table 1 depicts the material properties for the finite element modelling of the soft gripper.

In Figure $2 \mathrm{C}$, the mesh model consists of ten nodes of tetrahedral elements. Each element is $0.8 \mathrm{~mm}$ in size. A finite element analysis (ABAQUS) was carried out to analyse the finger's behaviour. As shown in Figure 2C, during simulation, the right side of the finger is constrained and the load applied is due to the pressure inside the air chamber. In the simulation, the static analysis is conducted and the effect of gravitation is taken into account. The size of the air chamber was changed so that the finger was able to move along a curving path while inflated by the air pressure, as shown in Figure 2C and D.

As shown in Figure 2C, three points on the model were selected to observe the displacement and the bending angle of the finger. Position 1 is located at the tip of the finger. When the finger inflated, it transformed itself into a curve. Position 2 is close to the middle of the finger; therefore, the deformation was relatively small compared to the first position. Position 3 hardly changed.

According to the results of the finite element analysis, the displacement and bending angle of all three positions were determined (as shown in Figure 3). The displacement was used to describe the movement of the finger, as it was measured from the $\mathrm{x}$-axis and y-axis. The three positions on the finger were selected to observe the behaviour of the finger as it curved up. The three points were selected at node of the finite element.

As the finger inflated, the displacement along with the bending angle was observed. The gripper was designed so that the tip of each finger bent inwards to grasp an object. Based on the results of the simulation, the size of the air chamber was modified to enable the finger to bend and curve up so as to handle an object.

In Figure 4, the experimental results and the simulation results indicate the bending angle of all three positions of the finger. Position1 appeared to be the most flexible when pressure was applied and was seen to bend the most.

\section{Pneumatic control system}

As shown in Figure 5A, the soft gripper consists of three fingers: each finger functions with the others through the connected controller and pneumatic regulator. The pneumatic regulator adjusts pressure and airflow through a normally-closed three-port solenoid valve (Burket 6014). When the gripper inflates, air inside the air chamber is pressurized. When the gripper deflates, air is released into the environment. The direction of the airflow is controlled by Arduino controller and force sensors.

Table 1. Material properties for the finite element modelling of the soft gripper.

$\begin{array}{lll}\text { Material } & \text { Density } & \text { Elasticity parameter } \\ \text { Sylgard } 186 & 1.12 \mathrm{~g} / \mathrm{cm}^{3} & \text { Hyperelastic (Mooney-Rivlin) } \\ & & \text { C10 }=1.13 \mathrm{E}-01 \mathrm{MPa} \\ & \mathrm{C}_{01}=2.83 \mathrm{E}-02 \mathrm{MPa} \\ & \mu=2.82 \mathrm{E}-01 \mathrm{MPa} \\ & \mathrm{E}=8.45 \mathrm{E}-01 \mathrm{MPa} \\ & \text { Poisson's ratio }=0.5 \\ & & \text { Elastic } \\ \text { Polycarbonate } & 1.20 \mathrm{~g} / \mathrm{cm}^{3} & \text { Young modulus }=2000 \mathrm{MPa} \\ & & \text { Poisson's ratio }=0.37 \\ & \end{array}$
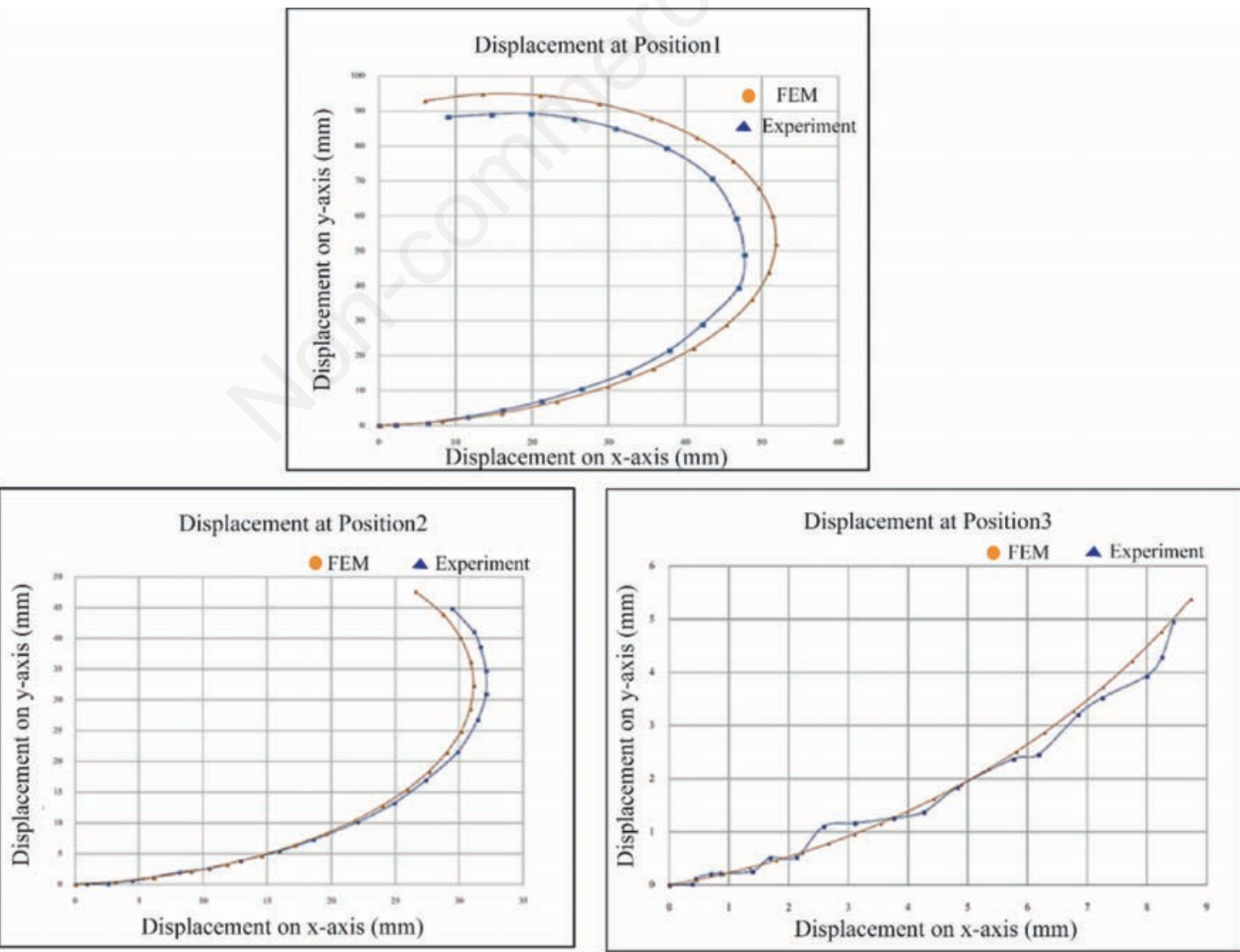

Figure 3. Experimental results and simulation results with displacement along the $\mathbf{x}$ and $\mathbf{y}$ axis. 
The contact force on the surface of a tomato is the normal force used to hold a tomato. The contact force has to be able to withhold the friction force between the gripper and the surface of the tomato. When a tomato ripens, its softness increases. To limit the gripping force, force sensors are attached to each finger. Based on the finite element simulation, the location of the force sensor is selected at the point where maximum force appears. The force sensor provides feedback, which is used to control the pressure inside the air chamber.
In Figure 5B, the value of $\mathrm{F}_{\text {limit }}$ is set-up to stop the released pressure from inflating the soft gripper. $F_{\max }$ is established as the maximum force that would not damage a tomato. $F_{\text {limit }}$ is set at $30 \%$ below the maximum force in order to prevent damage to the tomato. When the air chamber inflates, input pressure is increased until force feedback reaches $F_{\text {ref. Hence, }} F_{\text {ref }}$ is set at the minimum pressure to allow the gripper to hold the tomato. During this process, the $F_{\text {reading }}$ or reading force is continuously collected and compared to the limit force $\left(\mathrm{F}_{\text {limit }}\right)$. Since the pressure is released
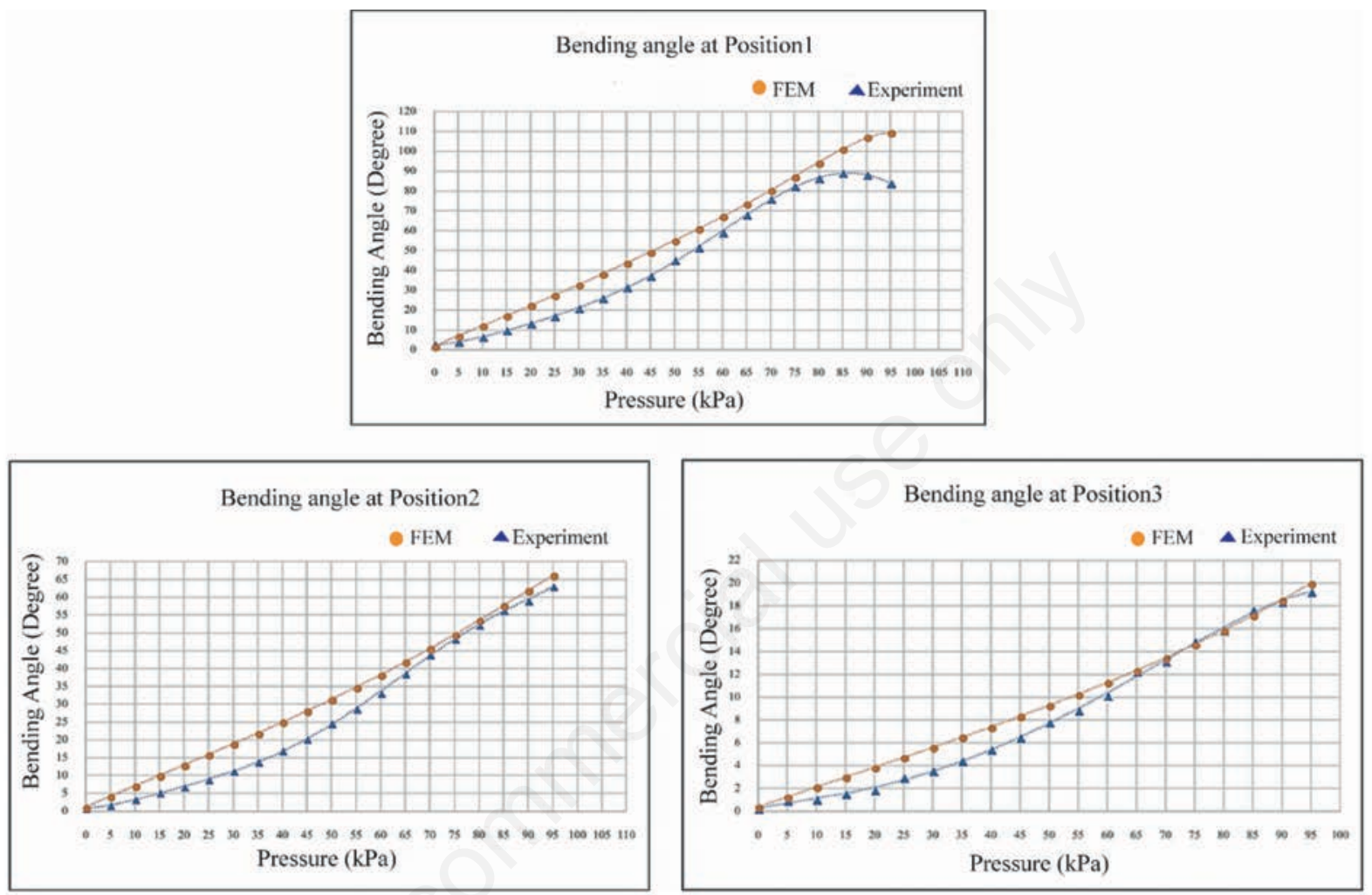

Figure 4. Experimental results and simulation results with the bending angle.
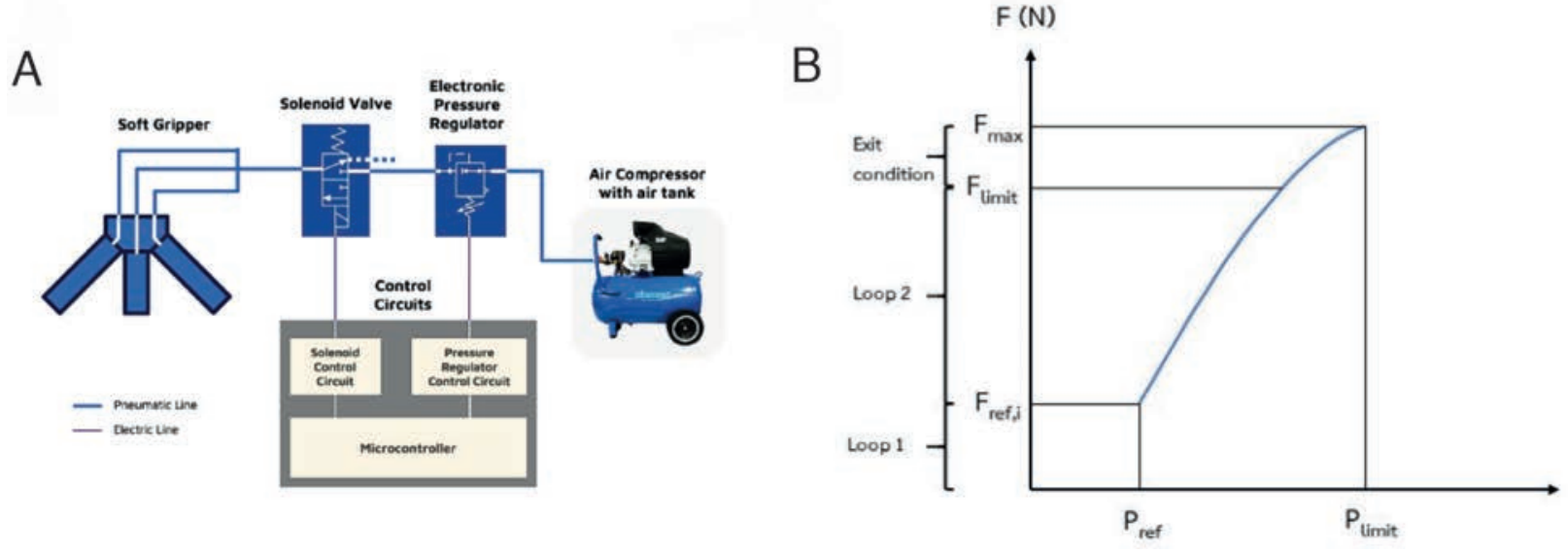

Figure 5. (A) Diagram of the control system; (B) Graph of force vs pressure of the finger based on the grasping test. 
into all three fingers simultaneously, the $F_{\text {reading for each finger is }}$ compared to the $\mathrm{F}_{\text {limit, }}$ one by one. When the difference between $F_{\text {reading }}$ and $F_{\text {limit }}$ is zero, the air pressure valve is turned off.

\section{Results}

\section{Finger test}

Each finger was tested for displacement and bending angle. The experimental results were compared with the results from the finite element simulation. It was noted that, at high pressure, the displacement error for all three points on the fingers was relatively low (within 10\%). This error must have occurred due to the pressure, when the finger inflated. When pressure was high, the finger proved hard to move. The actual displacement, therefore of all three points showed to be quite close to the results of the simulation.

\section{Grasping test}

To test the force of the gripper, a tomato was held by a threefingered soft gripper, applying force sensors (Interlink FSR400). During the experiment, the force sensors were placed along the finger at $19.4 \mathrm{~mm}, 32 \mathrm{~mm}$, and $47 \mathrm{~mm}$ respectively. Their locations were selected based on the results of the finite element simulation, where the stress appeared highly concentrated.

In Figure 6, when the finger inflated, pressure increased, and so did the gripping force. Therefore, the force, which was applied at various locations on the finger, was found to differ until the inside pressure reached a steady-state. At steady-state, the force, which was measured at all locations, was found to be about the same throughout the finger. In Figure 6, the point located close to the knuckle $(19.4 \mathrm{~mm})$ and the tip area $(47 \mathrm{~mm})$ were relatively the same for finger\#1 $(0.57 \mathrm{~N})$. A similar behaviour was observed in finger\#2 and finger\#3, where force sensors were placed at different locations: finger $\# 2$ at $47 \mathrm{~mm}$ and finger $\# 3$ at $19.4 \mathrm{~mm}$. In all three points on the finger, pressure increased, when the finger inflated. Subsequently, at a holding pressure of $70 \mathrm{kPa}$, the maximum force appeared at the tip of the finger. However, it may be that the lab prototype was not uniformly layered, therefore a different pressure might be applied to actuate all three fingers.

In Figure 7, the gripping position of each finger is shown. All three fingers are identical. The position of each finger was ascertained based on the position of the tomato, which hung downwards. Usually with two fingers on each side, the force required to hold the tomato is mostly be the friction force. When three fingers are used, the bottom finger helps to hold the tomato in place and prevents it from slipping. The gripper is designed to hold onto the tomato so the cutting mechanism can move closer to the plant to cut its stem. When the tomato is held, the recorded contact forces had a diameter of $79.03 \mathrm{~mm}$ with a weight of the tomato equal to $226 \mathrm{~g}$; for a diameter of $85.23 \mathrm{~mm}$, the tomato weighed $266 \mathrm{~g}$. When the holding pressure was applied, the force feedback was analysed and referred back to the controller in order to pressurize or depressurize the system.

Throughout the experiment, the maximum holding pressure applied to the grasping of the tomato was $70 \mathrm{kPa}$. As shown in Figure 7, the soft gripper was able to grip and hold the tomato successfully. Then, the gripper was attached to the one degree of freedom robot arm. As a result, the system became thoroughly integrated and the cutter was able to cut the stem of the tomato.

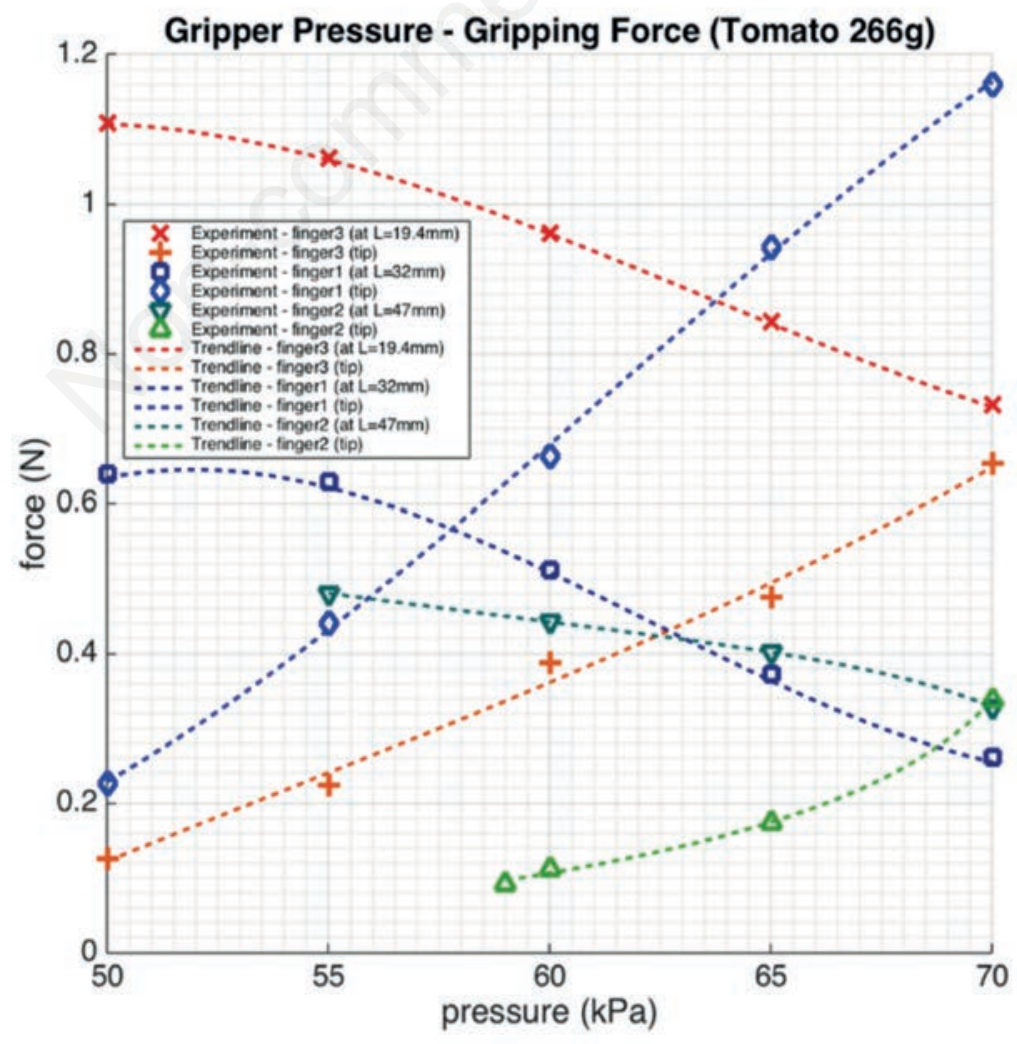

Figure 6. The relationship between the applied pressure and the force of the tip of the soft gripper, while grasping a $266 \mathrm{~g}$ tomato. 

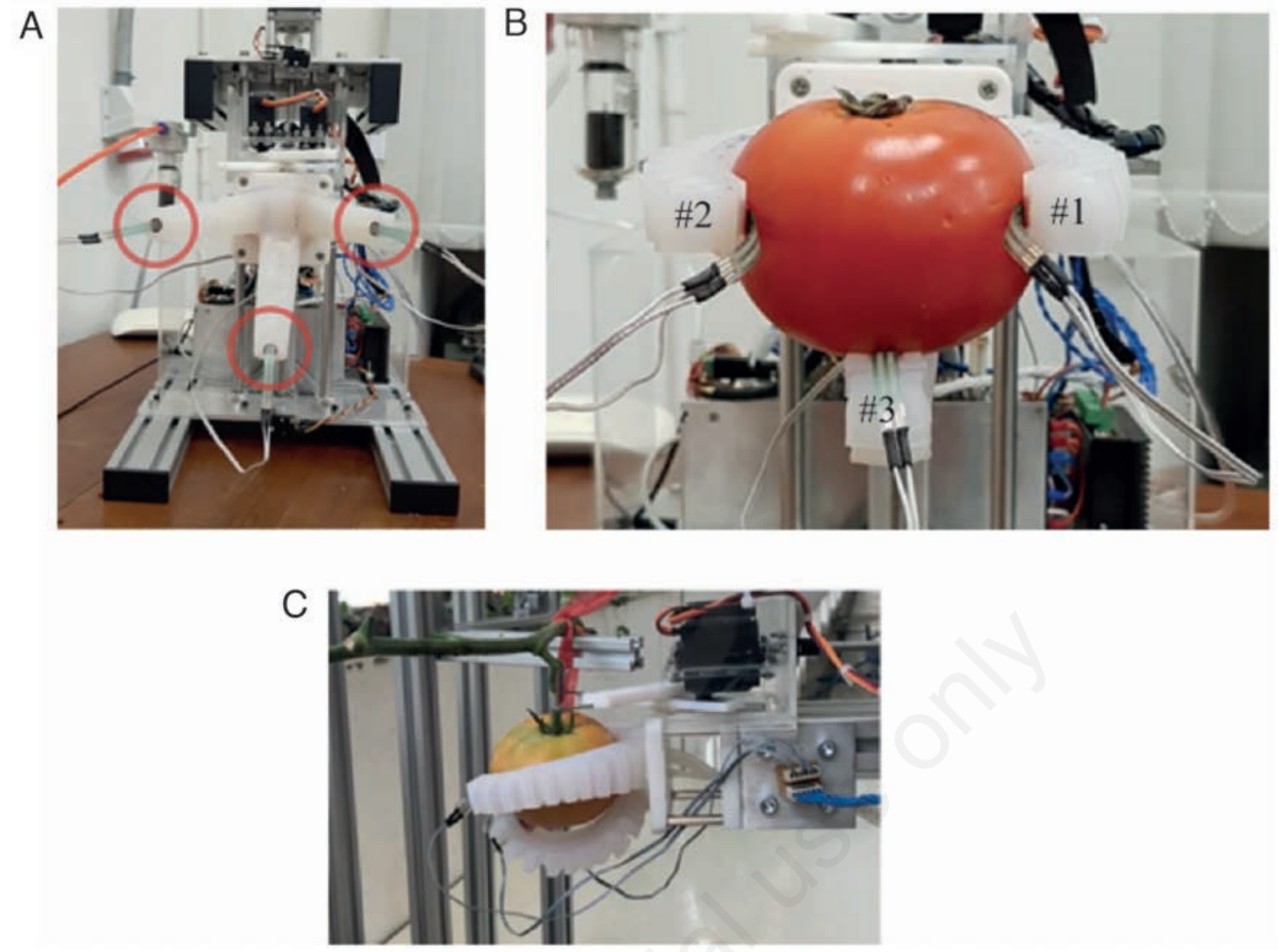

Figure 7. The position of the force sensor and the gripper during the tomato cutting process.

\section{Conclusions}

In this paper, a prototype for the application of a soft gripper in the field of agriculture is presented. A soft gripper can be used to harvest delicate fruits and vegetables, such as tomatoes. The gripper is actuated by applying a maximum air pressure of $95 \mathrm{kPa}$. To verify the performance of the three fingers during grasping, the relationship between force and air pressure was analysed. Then, also displacement and bending angles were examined as part of the design process. It is meaningful that the actual movement and bending angle of the real finger $v s$ the simulation results showed the following error range: $(0.04$ to $89.05 \%)$ for displacement and $(0.08$ to $57.88 \%)$ for the bending angle. The maximum force on each finger proved to be $1.62 \mathrm{~N}$, which was less than the tomato bio-yield of $2.57 \mathrm{~N}$. Therefore, the soft gripper applied a force that did not damage the tomatoes. This work describes the design of a soft gripper which proved able to harvest tomatoes quite successfully. Herein, the soft gripper shows its worth as an efficient and valuable farming instrument.

\section{References}

Bernardi L., Hopf R., Ferrari A., Ehret A.E., Mazza E. 2017. On the large strain deformation behavior of silicone-based elastomers for biomedical applications. Polym. Test. 58:189-98.

Brown E., Rodenberg N., Amend J., Mozeika A., Steltz E., Zakin M. R., Lipson H., Jaeger H.M. 2010. Universal robotic gripper based on the jamming of granular material. Proc. Natl. Acad.
Sci. 107:18809-14.

Hao Y., Liu Z., Liu J., Fang X., Fang B., Nie S., Guan Y., Sun F., Wang T., Wen L. 2020. A soft gripper with programmable effective length, tactile and curvature sensory feedback. Smart Mater. Struct. 29:035006.

Hayashi S., Ganno K., Ishii Y., Tanaka I. 2002. Robotic harvesting system for eggplants. JARQ. 36:163-68.

Ji C., Zhang J., Yuan T. , Li W. 2014. Research on key technology of truss tomato harvesting robot in greenhouse. Appl. Mech. Mater. 442:480-86.

Jinliang G., Zhao D., Wei J., Wu X. 2010. Design and control of the open apple-picking-robot manipulator. pp 5-8 in $3^{\text {rd }}$ Int Conf. Comp. Sci. Info. Tech., Chengdu, China.

Lehnert C., English A., McCool C., Tow A.W., Perez T. 2017. Autonomous sweet pepper harvesting for protected cropping systems. IEEE Rob. Autom. Lett. 2:872-9.

Liu J., Li P., Li Z. 2007. A multi-sensory end-effector for spherical fruit harvesting robot. pp 258-62 in IEEE Int. Conf. Autom. Logist., Jinan, China.

Liu J., Li Z., Wang F., Li P., Xi N. 2013. Hand-arm coordination for a tomato harvesting robot based on commercial manipulator. pp 2716-20 in IEEE Int. Conf. Rob. Biomimetics, Shenzhen, China.

Monta M., Kondo N., Ting K.C. 1998. End-effectors for tomato harvesting robot. J. Artif. Intell. Rev. 12:11-25.

Mosadegh B., Polygerinos P., Keplinger C., Wennstedt S., Shepherd R.F., Gupta U., Shim J., Bertoldi K., Walsh C.J., Whitesides G.M. 2014. Soft robotics: pneumatic networks for soft robotics that actuate rapidly. Adv. Funct. Mater 24:2109. 
Sirisomboon P., Tanaka M., Kojima T. 2012. Evaluation of tomato textural mechanical properties. J. Food Eng. 111:618-24.

Truby R.L., Wehner M., Grosskopf A.K., Vogt D.M., Uzel S.G.M., Wood R.J., Lewis J.A. 2018. Soft somatosensitive actuators via embedded 3D printing. Adv. Mater. 30:1706383.

van Henten E.J., Hemming J., van Tuijl B.A.J., Kornet J.G., Meuleman J., Bontsema J., van Os E.A. 2002. An autonomous robot for harvesting cucumbers in greenhouses. Auton. Rob. 13:241-58.

Wang G., Yu Y., Feng Q. 2016. Design of end-effector for tomato robotic harvesting. IFAC-Papers OnLine 49:190-3.

Wang Z., Zhu M., Kawamura S., Hirai S. 2017. Comparison of different soft grippers for lunch box packaging. Rob. Biomimet. 4:10. 